\title{
Empathy and Burnout in Medicine-Acknowledging Risks and Opportunities
}

\author{
Rajvinder Samra, PhD, MSc, BSC \\ School of Health, Wellbeing and Social Care, The Open University, Milton Keynes, UK.
}

J Gen Intern Med 33(7):991-3

DOI: $10.1007 / \mathrm{s} 11606-018-4443-5$

(c) Society of General Internal Medicine 2018

$\mathrm{P}$ atient-centeredness has been identified by the Institute of Medicine as one of the six main aims for a twenty-first century health system and encompasses qualities of compassion and empathy, as well as being responsive to the patients' values, needs and preferences. ${ }^{1}$ These tenets of modern medical professionalism require greater expectations over physicians' emotional availability than in the past, but this trend is risky in a context in which physicians show increasing rates of emotional vulnerability. ${ }^{2,3}$ A systematic review of 54 studies found between 20.9 to $43.2 \%$ of resident physicians report depression or depressive symptoms. ${ }^{3}$ Longitudinal studies indicate that the prevalence of depressive symptoms increases with increasing years in training, ${ }^{3}$ rather than diminishing with greater expertise and experience. A national survey found that $46 \%$ of practicing physicians show some signs of burnout, ${ }^{4}$ a phenomenon involving difficulties in dealing with stressful work and demands. Burnout can lead to feelings of depersonalisation and hopelessness ${ }^{2}$ and is associated with increased physician errors and reduced empathy for patients. ${ }^{4}$ It is therefore a concern that the demands and expectations on physicians' emotional resources are carefully balanced with the psychological costs and risks to their mental wellbeing in the provision of patient-centred empathic care.

Demonstrating empathy in clinical practice requires physicians to be able to listen to and understand the patient's perspective, communicate that understanding and act in a way that is helpful ${ }^{1}$ such as expressing sympathy, respect and support. The benefits to patients when physicians demonstrate clinical empathy are well reported, including greater patient satisfaction, openness about symptoms, reduced patient distress and improved outcomes. ${ }^{1}$ In contrast, low physician empathy is associated with burnout and emotional exhaustion, but the direction of the relationship is unclear. ${ }^{2}$ Demonstrating clinical empathy may contribute to compassion fatigue and emotional exhaustion which, in turn, leads to

Received March 8, 2018

Revised April 3, 2018

Accepted April 3, 2018

Published online April 16, 2018 physician burnout. ${ }^{2}$ The decline of empathy may be a coping mechanism for extreme emotional arousal; neuroscientific studies indicate that the brains of physicians show a downregulated empathic arousal response (e.g. negative affect or feelings) to witnessing distress compared to controls. ${ }^{2}$ Declining student empathy during medical school has been argued to represent the downregulation of empathy as a means of survival in the face of burnout or extreme distress. ${ }^{1}$ Competing time and resource demands and stressors in the environment have been identified as hindering emotional regulation, ${ }^{2}$ which may explain why offering empathic care can be emotionally exhausting for physicians - their ability to engage in emotional self-regulation is hindered or disrupted by the challenges of the modern medical workplace. Medical training should seek to better educate about, and mitigate against, the risks which may lead to reductions in empathy, or greater emotional vulnerability, to ensure that this does not become an enduring and worsening problem as the demands on medical students and physicians steadily increase.

There has been a general lack of acknowledgement in medicine of the social and biological variables that link empathy with gender role expectations and sex differences despite research evidence. Females are more likely to demonstrate empathy and it may be more socially acceptable for them to do so. ${ }^{5}$ Sex differences relating to empathy are consistent with neuroimaging studies which demonstrate greater neural activation in females during empathy tasks, findings from adolescent studies and arguments for the possible evolutionary roots for greater empathic concern in females. ${ }^{2}$ A 2013 study of 7584 board-certified practicing physicians found significant differences between males and females on a measure of emotional empathy. ${ }^{2}$ After controlling for age and experience, they found a highly specific effect of gender on 'empathic concern' (the tendency to experience feelings such as compassion and concern for others), but not on the component measuring cognitive empathy ('perspective taking': the capacity to understand another's experience or perspective). Female physicians reported higher levels of empathic concern, reported feeling less valued by patients and colleagues, and were more likely to report that their professional work had negatively affected their personal lives than males. The authors ${ }^{2}$ report that the higher rates of empathic concern in females corroborate research that suggests that women-in and outside of medicine - are more likely to report emotional exhaustion (depletion of emotional resources) $)^{5}$ than men. 
Emotional exhaustion is one of the three burnout dimensions along with reduced personal accomplishment (feelings of insufficiency and reduced professional self-esteem) and depersonalization (developing a callous cynical attitude). ${ }^{5}$

Burnout is a gradual process that develops over time; the order of presentation of the dimensions in the onset of burnout differs for men and women and, thus, the early warning signals are likely to differ. ${ }^{5}$ Houkes et al. ${ }^{5}$ collated findings from 18 studies from across the world incorporating samples from different occupational groups (including medicine) that reported significant gender differences in the prevalence and experience of burnout between males and females. They then conducted a three-wave longitudinal study of physicians using statistical modelling to explore the aetiology of burnout. ${ }^{5}$ They found evidence that the causal order of burnout dimensions differs between male and female physicians. The onset of male physicians' burnout presented with depersonalization, whereas female physicians' burnout was triggered by emotional exhaustion..$^{5}$ Additionally, for males only, their sense of personal accomplishment showed independence from the other two dimensions; males reported increasing personal accomplishment even as their emotional exhaustion and depersonalisation increased. This was not the case for females, whose burnout shows signs of reduction in their sense of personal accomplishment. Therefore, it is possible that reduced personal accomplishment is not a symptom of burnout for male physicians, but is for females. ${ }^{5}$ The authors ${ }^{5}$ recommend consideration of the differences in the dimensions and early presentation of burnout between male and female physicians should be incorporated in workplace training and policy development given the rapid feminization of the workforce.

Despite the evidence for differences in the experiences of empathy, as well as the burnout trajectory for male and female physicians, there appears to be limited consideration of these findings in the interventions to reduce burnout. Conceptually, two approaches of addressing the problem of declining empathy and increasing burnout in medicine have been identified: within the individual and within the environment. In terms of the individual, Gleichgerrcht and Decety ${ }^{2}$ recommend the optimal approach for fostering healthy clinical empathy in medical training is to focus on the cognitive components which concern understanding of another's experience and perspective, rather than the emotional component which concerns sharing in the emotions or feelings of another and may lead to emotional exhaustion. Issues related to differential experiences of empathy and different burnout trajectories, for males and females, are largely missing from the general recommendations for developing cognitive empathy or focusing on the cognitive components to empathy. Therefore, practical questions of when, where and how to intervene in medical education and training to address gender (societal expectations) and sex (biological) differences remain.

The American Medical Association (AMA) additionally recommends a systems-based approach to preventing physician burnout ${ }^{4}$ which marks a major leap forward in addressing the organisational and environmental factors that contribute to distress and burnout. The AMA Steps Forward: Preventing Physician Burnout is an online training module ${ }^{4}$ which encourages the development of a team-based model of care, with a focus on team-based communication, scheduling and infrastructure, such that patient visits may be team-based but physician-led. Typically, these systems-based approaches ${ }^{4}$ also fail to consider sex differences in experiencing empathy, gender differences relating to differential expectations towards physicians demonstrating empathic care, and ignores that the experience and trajectory of burnout differs may often differ for male and female physicians.

Addressing physician burnout is likely to require interventions that address how individual, dispositional factors (including sex differences) interact with physician's environment to produce conditions for burnout which may develop differently between male and female physicians. There is an opportunity to begin training in emotional regulation (empathy and avoiding burnout) during medical school and continue through residency training, on how situational/environmental stressors (workplace demands, time demands) deplete our psychological resources for emotional regulation. ${ }^{2}$ Medical education and training should prepare students and future physicians for possible differential experiences with regard to the emotional availability that might be expected of them, as women and men, from patients based on societal expectations. There is an opportunity to empower medical students and physicians in strategies for dealing with these possibly difficult patient encounters in order to mitigate against them should they occur. There exists a duty to further explore gender differences in how physicians might experience empathy and burnout, so that strategies for coping with the demands of emotional availability and vulnerability in the medical workplace may be more nuanced. Finally, there is an opportunity to train team members in the new team-based models of care that the experience of burnout, the burnout trajectory and the identification of burnout may have different characteristics and early warning signs for male and female physicians.

Contributors: RS is the sole author and contributor to this work.

Prior presentations: This work has not been previously presented elsewhere.

Corresponding Author: Rajvinder Samra, $\mathrm{PhD}, \mathrm{MSc}, \mathrm{BSc}$; School of Health, Wellbeing and Social Care, The Open University, Milton Keynes, UK (e-mail: Rajvinder.samra@open.ac.uk).

\section{Compliance with Ethical Standards:}

Conflict of Interest: The author declares he/she does not have a conflict of interest.

\section{REFERENCES}

1. Neumann M, Edelhäuser F, Tauschel D, et al. Empathy decline and its reasons: a systematic review of studies with medical students and residents. Acad Med. 2011;86(8):996-1009. 
2. Gleichgerrcht E, Decety J. Empathy in clinical practice: how individual dispositions, gender, and experience moderate empathic concern, burnout, and emotional distress in physicians. Plos One. 2013;8(4):e61526

3. Mata DA, Ramos MA, Bansal N, et al. Prevalence of depression and depressive symptoms among resident physicians: a systematic review and meta-analysis. JAMA. 2015;314(22):2373-83.
4. Linzer M, Guzman-Corrales L, Poplau, S. American Medical Association STEPS Forward: Preventing Physician Burnout. Available at: https://www stepsforward.org/modules/physician-burnout. Accessed 23 Mar 2018.

5. Houkes I, Winants Y, Twellaar M, Verdonk P. Development of burnout over time and the causal order of the three dimensions of burnout among male and female GPs. A three-wave panel study. BMC Public Health. 2011;11(1):240. 